\title{
Acceleration of ultrahigh-energy cosmic rays in the early afterglows of gamma-ray bursts
}

\author{
Ze-Lin Zhang, ${ }^{a, b, *}$ Ruo-Yu Liu ${ }^{a, b}$ and Xiang-Yu Wang ${ }^{a, b}$ \\ ${ }^{a}$ School of Astronomy and Space Science, Nanjing University \\ Xianlin Road 163, Nanjing 210023, China \\ ${ }^{b}$ Key laboratory of Modern Astronomy and Astrophysics (Nanjing University), Ministry of Education \\ Xianlin Road 163, Nanjing 210023, China \\ E-mail: zline355@foxmail.com, ryliu@nju.edu.cn, xywang@nju.edu.cn
}

\begin{abstract}
It has been suggested that ultrahigh-energy cosmic rays (UHECRs) can be produced by turbulent stochastic acceleration in relativistic jets of gamma-ray bursts (GRBs) on set of early afterglow. We develop a time-dependent model for proton energization by cascading compressible waves in GRB jets considering the concurrent effect of the jet's dynamics and the mutual interactions between turbulent waves and particles. Considering fast magnetosonic wave as the dominant particle scatterer and assuming interstellar medium (ISM) for the circumburst environment, our results suggest that protons can be accelerated up to $10^{19} \mathrm{eV}$ during the phase of early afterglow. The spectral slope $\mathrm{d} N / \mathrm{d} E \propto E^{0}$, which is consistent with the requirement for the performance of intermediate-mass composition of UHECR as measured by the Pierre Auger Observatory.
\end{abstract}

$37^{\text {th }}$ International Cosmic Ray Conference (ICRC 2021)

July 12th-23rd, 2021

Online - Berlin, Germany

\footnotetext{
${ }^{*}$ Presenter
} 


\section{Introduction}

The sites and mechanisms of the production of Ultrahigh-energy cosmic rays (UHECRs) are open questions. The study of the energy spectrum and the mass composition of UHECR helps to reveal their origin. Extragalactic sources, such as active galactic nuclei, Gamma-ray bursts (GRBs), energetic supernovae, tidal disruption events, galaxy clusters, as well as milli-second magnetars, have been considered as plausible candidates of UHECR sources.

GRBs have been studied extensively as the cosmic accelerator of UHECRs. The standard scenario adopted to produce non-thermal particles is the particle acceleration at shocks. However, particle acceleration by relativistic shocks with bulk Lorentz factor $\Gamma_{\mathrm{s}} \gg 1$ is limited by many factors. For example, the relative energy gain drops quickly (from $\Gamma_{\mathrm{s}}^{2}$ to $\simeq 2$ ) after the first shock crossing circle because of particles do not have sufficient time to become isotropic upstream before being caught up by the shock. Another possible disadvantage of the shock acceleration is the energy budget. The required energy production rate of CRs to explain the measured flux beyond the ankle is $10^{44} \mathrm{erg} \mathrm{Mpc}^{-3} \mathrm{yr}^{-1}$ while the gamma-ray energy production rate of GRBs is $10^{43} \mathrm{erg} \mathrm{Mpc}^{-3} \mathrm{yr}^{-1}$ for a typical gamma-ray luminosity of $10^{52} \mathrm{erg} \mathrm{s}^{-1}$ and a local GRB rate of $1 \mathrm{Gpc}^{-3} \mathrm{yr}^{-1}$. Given the predicted spectral slope of the accelerated particles being $p \gtrsim 2$ for the relativistic shock acceleration, the fraction of the energy of CRs accelerated beyond the ankle $\left(10^{18.5} \mathrm{eV}\right)$ is only at the level of $10 \%$ of the total CR energy. As a result, it would require a baryon loading factor (defined as the ratio of total energy populated in CRs to that in gamma rays) of $\sim 100$ to account for the required UHECR energy production rate. It is in tension with the constraint from the non-detection of GRB neutrinos by the IceCube neutrino telescopes in some dissipation mechanisms of GRBs. Furthermore, it has been pointed out that a very hard CR injection spectrum with $p \lesssim 1$ is favored in order to fit the spectrum and composition of UHECRs measured by the Pierre Auger Observatory, where the best-fit index is even $p<0$.

Recently, a stochastic acceleration (SA) model of UHECRs via turbulence in GRB jets has been proposed to avoid the problems mentioned above [1]. The SA can yield a hard UHECR spectrum with shallow index $p \lesssim 2$ [2]. Magnetohydrodynamic (MHD) turbulence is indispensable in various astrophysical processes. Particle scattering and diffusion largely rely on the properties of plasma turbulence. Fast modes waves show an isotropic cascade and it could be the most effective scatterers of cosmic-rays [3]. The spectrum of the isotropic cascade was claimed to be $k^{-3 / 2}$.

In this work, we consider the turbulence driven by MHD instabilities induced by the jet's propagation in the circumburst interstellar medium (ISM). The turbulence is injected at the scale comparable to the size of the shock and then cascades down to small scales due to the wave-wave interactions. Charged particles are expected to be accelerated by MHD waves under resonant condition $\omega-k_{\|} v_{\|}=l \Omega_{\mathrm{g}}(l=0, \pm 1, \pm 2, \ldots)$, where $\omega$ is the wave frequency, $k_{\|}$the parallel wavenumber, $v_{\mathrm{w}}$ the phase velocity, and $v_{\|}=\mu \nu$ the particle velocity parallel to the mean magnetic field $B \equiv|\boldsymbol{B}|$, and $\mu$ the pitch-angle cosine, $\Omega_{\mathrm{g}}$ the gyro-frequency of relativistic particles. In our work, we only consider the resonance occurring at $l=-1$.

In the previous work [1], the authors considered the SA process with the test-particle treatment and assume non-evolving parameters such as the particle injection rate and the diffusion coefficient. In fact, acceleration of particles consumes the turbulence energy, representing as a damping process. In the meantime, it relaxes the confinement of particles in the jet and may cause particle escape 
from the jet. In addition, the GRB jet gets decelerated as it expands into the ISM. As a result, relevant parameters for the SA process evolves with time and particles that confined in the jet suffer the adiabatic cooling. These processes have not been considered in the previous work but they may significantly affect the SA process, and, consequently, the accelerated CR spectrum.

In this work, we attempt to model the acceleration of UHECRs via the SA process in the early afterglow of GRBs with incorporating jet's dynamics and wave-particle gyroresonant interactions. The configuration of this work proceeds as follows. In Sec. 2, the gyro-resonant interaction of wave-particle by coupled kinetic equations in the early afterglows of GRBs is introduced. In Sec. 3, we analysis the acceleration of UHECR by wave-particle interactions in a comprehensive way which covers the behaviors of wave-particle spectra. Conclusions are presented in Sec. 4.

\section{Stochastic acceleration in the early afterglows of GRBs}

For an isotropic-equivalent, adiabatic GRB ejecta expanding in ISM [4], the following equations have been proposed to depict its dynamic evolution:

$$
\begin{array}{r}
\frac{\mathrm{d} \Gamma}{\mathrm{d} m} \simeq-\frac{\Gamma^{2}-1}{M_{\mathrm{ej}}+2 \Gamma m}, \\
\mathrm{~d} m=4 \pi R^{2} n_{\mathrm{ISM}} m_{\mathrm{p}} \mathrm{d} R, \\
\mathrm{~d} R=\beta_{\mathrm{sh}} c \Gamma\left(\Gamma+\sqrt{\Gamma^{2}-1}\right) \mathrm{d} t,
\end{array}
$$

where $\Gamma$ is the bulk Lorentz factor of the external shock, $m$ and $M_{\text {ej }}$ are the rest mass of the swept-up ISM and the mass ejected from the GRB central engine respectively. $R$ is the radius of the external shock, $n_{\mathrm{ISM}}$ the number density of the interstellar medium, $m_{\mathrm{p}}$ the mass of a proton, $\beta_{\mathrm{sh}}=v_{\mathrm{sh}} / c$ where $v_{\text {sh }}$ is the bulk velocity of the material and $c$ is the speed of light.

At the onset of the afterglows (external shocks) of GRBs, the relativistic outflowing plasma can excite large scale turbulences by MHD instabilities. Particles in plasmas scatter off the randomly moving induced-turbulence. After a period of "scattering", the transition from anisotropic particle velocity distribution to the isotropic one. In consideration of particle injection, escape and adiabatic energy loss processes, the evolution of the proton energy distribution $N(E, t)$ in the outflowing plasma (jet) comoving frame can be described by the Fokker-Planck (FP) equation [2]:

$$
\frac{\partial N}{\partial t}=\frac{\partial}{\partial E}\left[\mathcal{D}_{\mathrm{EE}}(E, t) \frac{\partial N}{\partial E}\right]-\frac{\partial}{\partial E}\left[\left(\frac{2 \mathcal{D}_{\mathrm{EE}}(E, t)}{E}+\langle\dot{E}\rangle\right) N\right]-\frac{N}{t_{\mathrm{esc}}}+Q_{\mathrm{inj}}(E, t),
$$

where $\langle\dot{E}\rangle=-E / t_{\mathrm{ad}}$ represents the adiabatic energy loss of relativistic expansion, $t_{\mathrm{ad}}=R /(\Gamma c)$ the adiabatic energy loss timescale. The last term $Q_{\mathrm{inj}}(E, t)=Q_{0}(t) \delta\left(E-E_{\mathrm{inj}}\right)$ represents the continuous particle injection from the initial moment, $Q_{0}(t)=4 \pi R^{2} \Gamma n_{\mathrm{ISM}} c$ the number density at the proton injection energy $E_{\text {inj }}$, and we are assuming continuous injection of particles at $E_{\text {inj }}=300 \Gamma_{300} m_{\mathrm{p}} c^{2}$ during the early afterglows evolution, $E=\Gamma m_{\mathrm{p}} c^{2}$ the proton energy. The term $-N / t_{\text {esc }}$ represents the spatial diffusive escape of the particle from the accelerated region the size of which is $R / \Gamma$ in the jet's comoving frame. The spatial diffusion coefficient $\mathcal{D}_{\mathrm{RR}}$ is related to the energy diffusion coefficient $\mathcal{D}_{\mathrm{EE}}$ by $\mathcal{D}_{\mathrm{RR}} \mathcal{D}_{\mathrm{EE}} \approx \beta_{\mathrm{w}}^{2} E^{2}$. Therefore, the escape time $t_{\mathrm{esc}} \approx R^{2} / \mathcal{D}_{\mathrm{RR}} \approx R^{2} /\left(\Gamma^{2} v_{\mathrm{w}}^{2} t_{\mathrm{acc}}\right)$, where $t_{\mathrm{acc}} \approx E^{2} / \mathcal{D}_{\mathrm{EE}}$ the acceleration time for protons whose Larmor radii resonate with some 
character length scales of the turbulent magnetic fields, $v_{\mathrm{w}}$ the phase speed of fast magneto-sonic waves. The cooling effects owing to photopion production and proton synchrotron radiation can be neglected [1].

Since we deal with ultra-relativstic particles, the particle velocity $v \gg v_{\mathrm{w}}$ is considered. Hence, we use an approximated form of the diffusion coefficient in energy space given by [5]:

$$
\mathcal{D}_{\mathrm{EE}}(E) \sim \frac{E^{2} \beta_{\mathrm{w}}^{2} k_{\mathrm{res}} c}{r_{\mathrm{g}} u_{\mathrm{B}}} \int_{k_{\mathrm{res}}}^{k_{\max }} k^{-1} W_{\mathrm{B}}(k) \mathrm{d} k
$$

where the dimensionless speed is given by:

$$
\beta_{\mathrm{w}}=\frac{v_{\mathrm{w}}}{c}=\sqrt{\frac{\hat{\gamma} P+B^{2} / 4 \pi}{\rho c^{2}+\hat{\gamma} P /(\hat{\gamma}-1)+B^{2} / 4 \pi}},
$$

and $\hat{\gamma}=4 / 3$ represents the adiabatic index in the relativistic regime, $P=\left(4 \Gamma^{2} n_{\mathrm{ISM}} m_{\mathrm{p}} c^{2}\right) / 3$ the relativistic gas pressure, $\rho=4 \Gamma n_{\mathrm{ISM}} m_{\mathrm{p}}$ the downstream rest mass energy density, and $n_{\mathrm{ISM}}$ the upstream rest number density of protons. The mean magnetic field energy density satisfying

$$
u_{\mathrm{B}}=\frac{B^{2}}{8 \pi}=\int_{k_{\min }}^{k_{\max }} W_{\mathrm{B}}(k) \mathrm{d} k
$$

with the assumption of the initial magnetic field $B_{0} \simeq\left(32 \pi \varepsilon_{\mathrm{B}} \Gamma_{0}^{2} n_{\mathrm{ISM}} m_{\mathrm{p}} c^{2}\right)^{1 / 2}$, and $\varepsilon_{\mathrm{B}}$ the magnetic field equipartition factor. $W_{\mathrm{B}}(k) \approx W(k) / 2$ being the magnetic component of $W(k)$ (the total energy density of the turbulent fields per unit wavenumber). $k_{\text {res }} \equiv 1 / r_{\mathrm{g}}(E)$ is the corresponding wavenumber of the wave resonating with protons of energy $E$, where $r_{\mathrm{g}} \simeq E /(e B)$ is the Larmor radius of the protons. $k_{\min }=2 \pi / \lambda_{\text {inj }}$ and $k_{\max }=2 \pi / \lambda_{\min }$ with $\lambda_{\min }$ the smallest eddy scale and $\lambda_{\text {inj }}$ the injection eddy scale. Hence, we use a dimensionless parameter $\xi$ to parametrize $\lambda_{\text {inj }}=\xi R / \Gamma \lesssim R / \Gamma$. The value of $\lambda_{\max }$ is rather trivial, i.e., $2 \pi r_{\mathrm{g}}\left(E_{0}\right)$.

The gyro-resonant interactions lead to energy exchange between the turbulent waves and particles. As we mentioned above, the fast mode waves in relativistic jets are taken to be isotropic, and their spectral density $W(k, t)$ in wavenumber space is determined by the FP equation [6]:

$$
\frac{\partial W}{\partial t}=\frac{\partial}{\partial k}\left[\mathcal{D}_{\mathrm{kk}}(k, t) \frac{\partial W}{\partial k}\right]-\frac{\partial}{\partial k}\left[\frac{2 \mathcal{D}_{\mathrm{kk}}(k, t)}{k} W\right]+\frac{k}{3}(\nabla \cdot \mathbf{v}) \frac{\partial W}{\partial k}+\Gamma_{\mathrm{w}}(k, t) W+Q_{\mathrm{w}, \text { inj }}(k, t),
$$

where the third term on the RHS represents the energy loss of adiabatic expansion of magnetic fields at different scales and $\mathbf{v}$ the expansion velocity of the waves, $\Gamma_{\mathrm{w}}(k, t)$ represents the damping effect, and $Q_{\mathrm{w}, \text { inj }}(k, t)=Q_{\mathrm{w} 0}(t) \delta\left(k-k_{\text {inj }}\right)$ represents the continuous energy injection into the turbulence at a mono-scale $\lambda_{\text {inj }}=1 / k_{\text {inj }}$, where $Q_{\mathrm{w} 0}=4 \Gamma^{2} \varepsilon_{\mathrm{B}} n_{\mathrm{ISM}} m_{\mathrm{p}} c^{2} /(R / \Gamma c)$ the injection rate per unit volume at the wavenumber $k_{\text {inj }}$. Note that $k_{\text {inj }}$ is not to be confused with another characteristic wavenumber

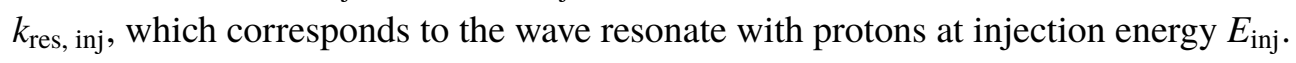

Considering the compressible fast mode waves with the Iroshnikov-Kraichnan-type (IK-type) turbulence, and the diffusion coefficient in wavenumber space $\mathcal{D}_{\mathrm{kk}}(k)$ can be given by [6]:

$$
\mathcal{D}_{\mathrm{kk}}(k)=C^{2} k^{4} v_{\mathrm{w}}\left[\frac{W(k)}{2 u_{\mathrm{B}}}\right]
$$

where $C$ is the IK constant of order unity. 
During the prompt emission phase, we assume an initial condition for $W(k, t)$ as

$$
W(k, t=0) \equiv \kappa_{0} u_{\mathrm{B}}\left(\frac{k}{k_{\mathrm{inj}}}\right)^{-q} \exp \left(-\frac{k}{k_{\max }}\right)
$$

where the parameter $\kappa_{0} \approx-2 k_{\text {inj }}^{q}\left(k_{\text {max }}^{-q+1}-k_{\text {inj }}^{-q+1}\right), u_{\mathrm{B}}=4 \Gamma^{2} \varepsilon_{\mathrm{B}} n_{\mathrm{ISM}} m_{\mathrm{p}} c^{2}$ the comoving magnetic energy density, and the IK-type spectral index $q=3 / 2$.

The energy gain of particles serves as a damping process for the turbulence. We here only consider the damping of the turbulence due to the gyro-resonance of protons. Therefore, the energy dissipation rate of the turbublence should be equal to the energy gain rate of the protons, i.e.,

$$
\int \mathrm{d} k \Gamma_{\mathrm{w}}(k) W(k)=-\int \mathrm{d} E E \frac{\partial}{\partial E}\left[E^{2} \mathcal{D}_{\mathrm{EE}}(E) \frac{\partial}{\partial E} \frac{N(E)}{E^{2}}\right] .
$$

From Eq. (5), integrating by parts twice, we obtain

$$
\Gamma_{\mathrm{w}}(k)=-\frac{4 \pi e^{2} \beta_{\mathrm{w}}^{2} c}{k}\left[N\left(E_{\mathrm{res}}(k)\right)+\int_{E_{\mathrm{res}}(k)}^{E_{\mathrm{max}}} \frac{2 N(E)}{E} \mathrm{~d} E\right] .
$$

The turbulence at the wavenumber $k$ is damped by protons with energy $E>E_{\text {res }}(k)$ where $E_{\text {res }}=$ $e B / k$. The turbulent magnetic fields in the relativistic jet indicate $\delta B \lesssim B$.

\section{Results and discussions of turbulent stochastic acceleration}

We adopt the Ronge-Kutta method to solve the dynamical evolution of the GRB jet, and the central difference method to solve the time-dependent FP equations, see details in the Appendix of Ref. [7]. UHECR protons accelerated by turbulence through wave-particle gyro-resonant interactions are considered under two different cases of $\xi=0.1$, "I" for $n_{\mathrm{ISM}}=0.01 \mathrm{~cm}^{-3}$ and "II" for $n_{\mathrm{ISM}}=1 \mathrm{~cm}^{-3}$. Both cases take the initial bulk Lorentz factor $\Gamma_{0}=300 \Gamma_{300}$ and consider the onset of the afterglow at $0.1 \mathrm{~s}$ (in the observer's frame) after the burst so that the initial radius of the early afterglows are set to $R_{0} \simeq 2 \Gamma_{0}^{2} c t_{0}=5.4 \times 10^{14} \mathrm{~cm}$. We then evolve the system for a total time $t_{\mathrm{cmv}}=40000 \mathrm{~s}$ in jet's comoving frame. The time-dependent proton spectra are shown in the upper panels of Fig. 1, where some relevant timescales are shown in the lower panels.

Considering the adiabatic cooling slightly softens the spectrum at the cutoff regime (where $t_{\text {acc }} \simeq t_{\text {ad }}$ or $E \simeq E_{\text {eq }}$ ) as shown with the thin dashed lines. Diffusive escape of particles does not have significant influence on the spectrum at the high-energy end, but play an important role in shaping the spectrum around $E_{\mathrm{inj}}$, as shown in the upper panels of Fig. 1. This can be also seen by comparing the timescales shown in the lower panels in Fig. 1. At the high energy end, when the acceleration timescale becomes comparable to the adiabatic cooling timescale (which is also comparable to the dynamical timescale), the diffusive escape timescale is still several times longer. From Fig. 1, we can see that the influence of the adiabatic cooling effect to the spectrum of the proton is not significant. As shown in the top panels of Fig. 1, it is worth noting that, the total kinetic energy of protons at injection is $E_{\mathrm{tot}} \sim \Gamma^{2} M_{\mathrm{sw}} c^{2} \sim 10^{54}$ ergs where $M_{\mathrm{sw}}$ is the mass of swept-up material, but protons accelerated up to the high-energy end via extracting the turbulent magnetic field energy is well limited by the magnetic equipartition factor $\varepsilon_{B}=0.05$. Hence, the baryon loading factor of accelerated protons is naturally determined instead of manual operation. 
(a)

(a) $t_{\text {obs }}$

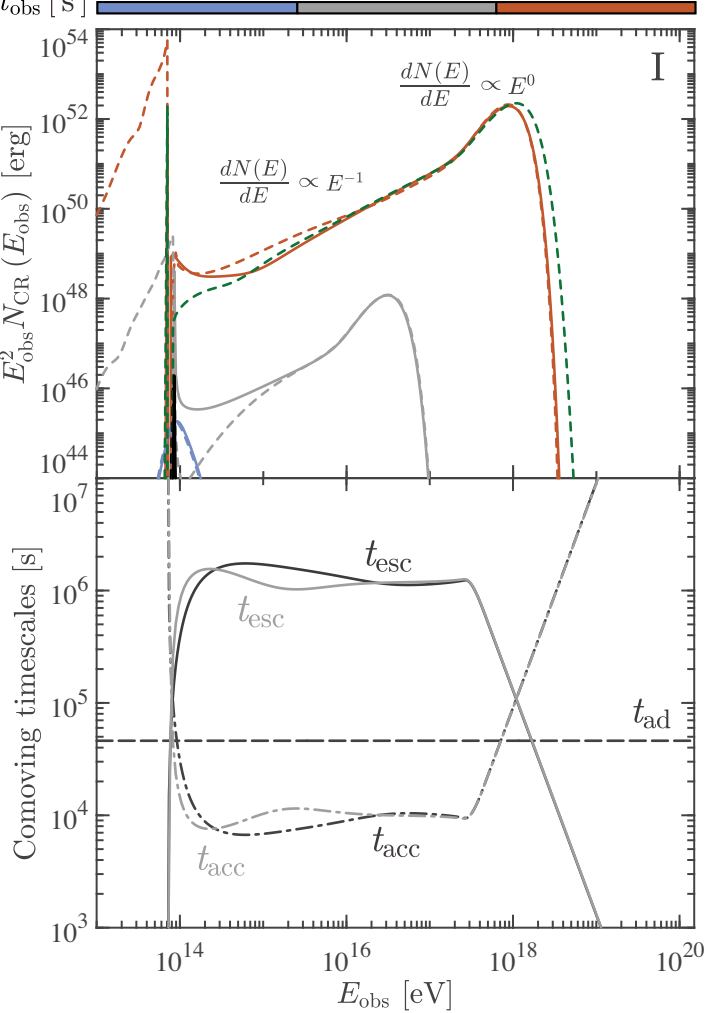

(b)

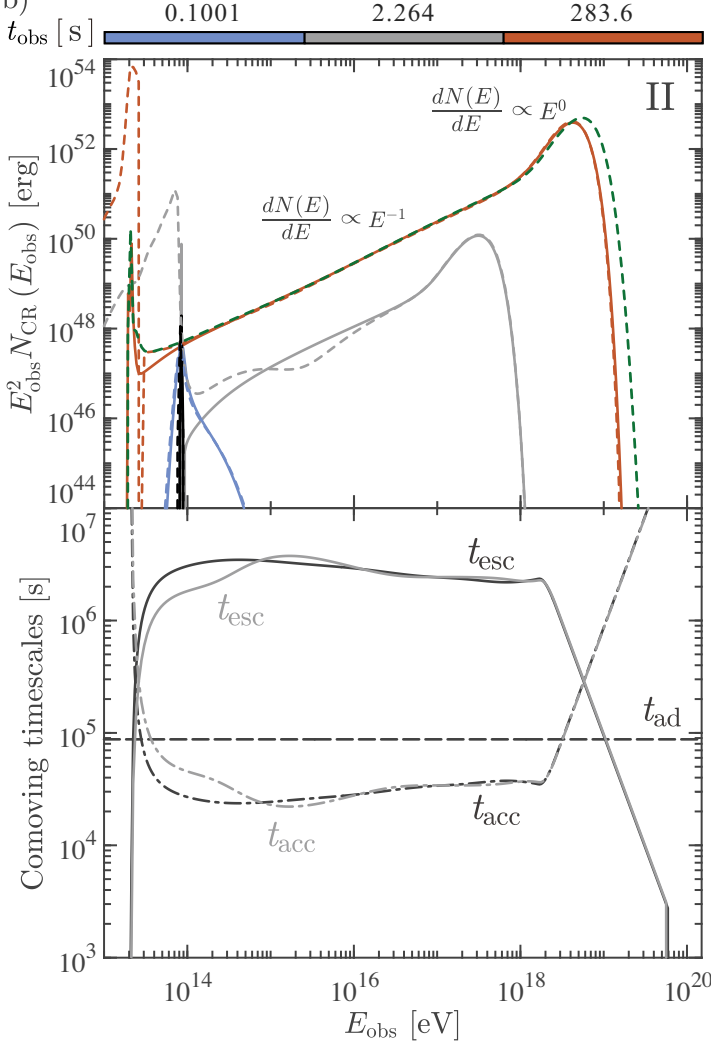

Figure 1: UHECR protons spectra resulting from joint stochastic acceleration, particle diffusive escape, and adiabatic energy losses and their corresponding comoving timescales as a function of observed proton energy. Upper panels: The black solid and dashed lines represent proton injection at $0.1000 \mathrm{~s}$ in the observer's frame which is not presented on the colorbar. The corresponding same color short-dashed lines depict the evolution without considering the particle spatial diffusive escape effect. The green short-dashed lines in the upper panels delineate the case ignoring the adiabatic energy loss at the final moment. Lower panels: Comoving timescales against observed proton energy under the case of $\xi=0.1$. The evolution of the energy spectra of protons for $40000 \mathrm{~s}$ in the comoving frame of relativistic outflowing plasma with $n_{\text {ISM }}=0.01 \mathrm{~cm}^{-3}$ (case I) and $n_{\mathrm{ISM}}=1 \mathrm{~cm}^{-3}$ (case II). The acceleration, adiabatic expansion cooling and diffusive escape timescales are separately denoted by dash-dotted, dashed and solid lines. The gray lines show the evolution without including adiabatic loss process. Proton spectra evolve during: (a) $t_{\mathrm{obs}} \in[0.1001 \mathrm{~s}, 80.35 \mathrm{~s}]$ and (b) $t_{\mathrm{obs}} \in[0.1001 \mathrm{~s}, 283.6 \mathrm{~s}]$ in the observer's frame, respectively. We only show the final moment of different timescales in the lower panels.

According to Ref. [2], if $q=3 / 2$, the steady-state particle spectrum implied by Eq. (4) is $\mathrm{d} N / \mathrm{d} E \propto E^{1-q}$ when $E \in\left(E_{\mathrm{inj}}, E_{\mathrm{eq}}\right)$, as long as the particle escape can be neglected $\left(t_{\mathrm{esc}} \gg t_{\mathrm{acc}}\right.$, $\left.t_{\mathrm{ad}}\right)$. So the power-law energy spectra $E^{2} N_{\mathrm{CR}}(E)$ is proportional to $E^{3 / 2}$. This is the result obtained in the test particle limit and without considering the dynamic evolution of the system. From Fig. 1, we see that the bulk of the accelerated particle spectra in both considered cases are softer. In general, when taking into account the feedback of particle acceleration on the turbulence, the turbulence energy is consumed. Such a negative feedback from the protons impedes themselves to be further accelerated. It is interesting to note that many previous literature found a very small $\varepsilon_{\mathrm{B}}$ for the 

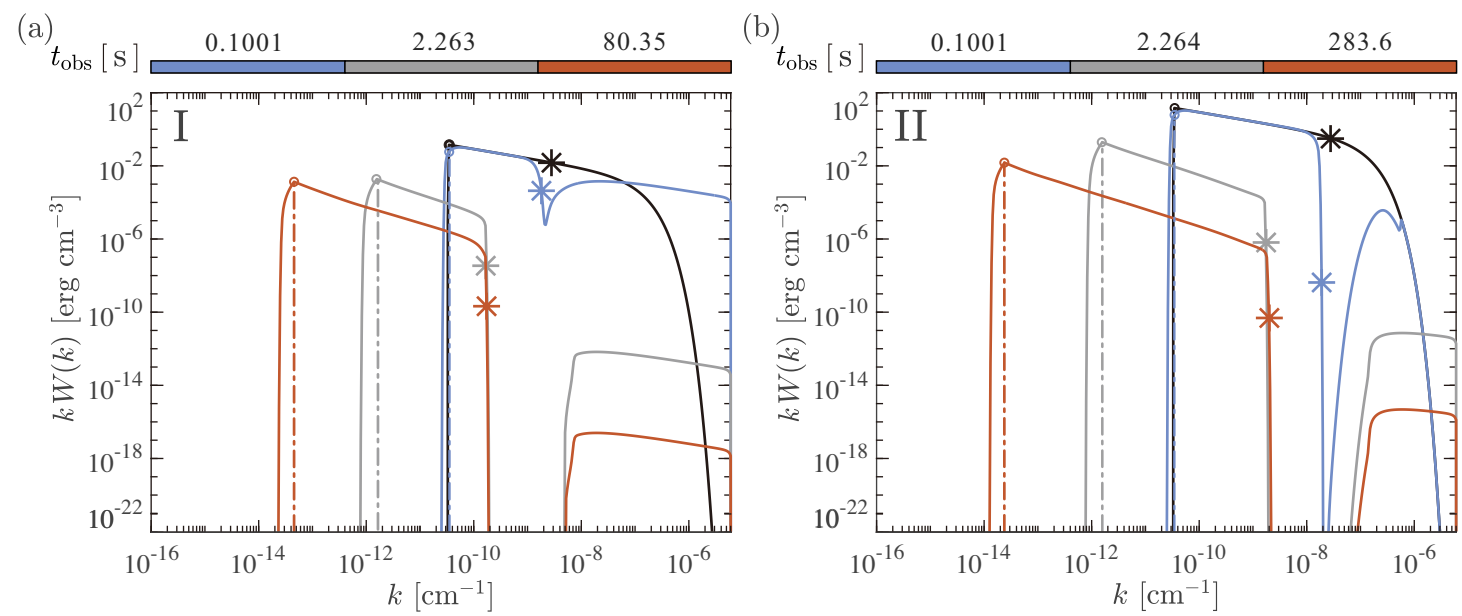

Figure 2: Turbulence spectral energy density against wavenumber. The initial wave energy (magnetic field) injection at $0.1000 \mathrm{~s}$ in observer's frame are separately represented by the black solid lines are not shown on the colorbar. The solid lines lines from blue to gray then to red represent the evolution of the turbulent waves. The evolution of the relativistic outflowing plasma wave spectra for $t_{\mathrm{cmv}}=40000 \mathrm{~s}$ with $n_{\mathrm{ISM}}=0.01 \mathrm{~cm}^{-3}$ (case I) and $n_{\mathrm{ISM}}=1 \mathrm{~cm}^{-3}$ (case III). Evolution time: (a) $t_{\mathrm{obs}} \in[0.1001 \mathrm{~s}, 80.35 \mathrm{~s}]$ and (b) $t_{\mathrm{obs}} \in[0.1001 \mathrm{~s}, 283.6 \mathrm{~s}]$ in the observer's frame, respectively. Asterisks on the lines show the turbulent magnetic field energy density which are corresponding to the wavenumber $k_{\text {res, inj. The circles at different }}$ moments show the injected position of turbulent waves. The dash-dotted lines represent the cut-off position of injection wavenumber at different moments.

external shock when modelling the multiwavelength afterglow of some GRBs (e.g., Ref.[8]), which significantly deviates from the energy-equipartition value. We speculate that the feedback of the particle acceleration on the turbulence energy could be a reason. This will be studied elsewhere.

In the meanwhile, the magnetic energy is also lost due to the adiabatic expansion of the jet. Since we assume the injection eddy size to be proportional to the jet's radius, the expansion of the jet also reduces the injection wavenumber of the turbulence $k_{\text {inj. }}$. The turbulent energy would then distribute over a larger and larger range in the wavenumber space, so that the energy density per wavenumber is reduced. Therefore, compared to the case in the test particle limit and the steady state, there will be a decline in the capacity of the stochastic acceleration with time. This is also reflected in the particle spectrum. We can see the bulk of the accelerated particle spectrum is softer than $E^{3 / 2}$.

According to above parameters evolution, the shape of the wave energy density spectra can be easily settled down from two types of wavenumber, $k_{\text {inj }}$ and $k_{\text {res, inj, }}$, as shown in Fig. 2. As the turbulent eddy scale becomes larger and larger, the wavenumber of the it becomes smaller and smaller. The larger wavenumber associated eddies (smaller scale) have already been damped by the corresponding lower energy particles, the relative higher energy particles trapped in the acceleration region which can continuously gain energy from the lower wavenumber turbulent waves. Then energy transport in $k$-space will cause more remarkable deviation from the IK-type spectrum in lower wavenumber.

In the case of ISM environment around bursts, our results suggest that a combination of cyclotron wave damping and gyro-resonant particle acceleration in the early afterglows of GRBs 
could account for the origin of UHECRs. It is worth noting that the evolution of jet's dynamics can reduce the acceleration capacity of turbulence due to the persistent consumption of magnetic fields by protons energization. In other words, the fluctuated magnetic field can energize cosmic rays more efficiently without considering the evolution of jet's dynamics.

\section{Conclusions}

In this paper, we take into account the concurrence of GRBs jet's dynamics and the kinetic descriptions of wave-particle interactions including SA process of particles and the damping of MHD fast-mode waves. Protons can be accelerated to ultrahigh-energy by turbulent waves through wave-particle gyro-resonant interactions.

Including the evolution of jet's dynamics can reduce the energy density of the turbulent magnetic fields, and subsequently weaken the capacity of the acceleration of the SA mechanism. Since energies of accelerated particles originate from the magnetic turbulence, taking into account the feedback (i.e., damping) of particle acceleration on the turbulence spectrum leads to a weaker magnetic field compared to that predicted in the standard afterglow dynamic model, given that the magnetic energy is consumed by particles. It also results in a particle spectrum softer than that predicted in the test-particle limit. Considering the fast mode of magnetosonic wave as the dominant particle scatterer and assuming ISM for the circumburst environment, we found that protons can nevertheless be accelerated up to $10^{19} \mathrm{eV}$ with a spectrum $\mathrm{d} N / \mathrm{d} E \propto E^{-1}$ for some favorable choices of system's parameters. We also found that a pile-up bump may occur in the spectrum ahead of the cutoff, leading to a very hard particle spectrum with $\mathrm{d} N / \mathrm{d} E \propto E^{0}$. On the other hand, the maximum energy (or cutoff energy) of the accelerated protons is reduced because the maximum achievable energy in the acceleration is limited by the eddy scale.

Compared to the traditional acceleration model by relativistic shocks, our model not only alleviates the energy budget problem, but also provide a mechanism to generate the hard injection spectrum as required by explaining the measured UHECRs spectra above the ankle and the chemical composition of UHECR as measured by the Pierre Auger Observatory.

\section{References}

[1] K. Asano, and P. Mészáros, Phys. Rev. D 94, 023005 (2016).

[2] Ł. Stawarz, and V. Petrosian, Astrophys. J. 681, 1725 (2008).

[3] K. D. Makwana, and H. Yan, Phys. Rev. X 10, 031021 (2020).

[4] Y. F. Huang et al., Mon. Not. R. Astron. Soc. 309, 513 (1999).

[5] J. Kakuwa, Astrophys. J. 816, 24 (2016).

[6] J. A. Miller et al., Astrophys. J. 461, 445 (1996).

[7] R. Y. Liu, F. M. Rieger, and F. A. Aharonian, Astrophys. J. 842, 39 (2017).

[8] R. Y. Liu, and X. Y. Wang, Astrophys. J. 730, 1 (2011). 